\title{
A 36-year-old man with hemoptysis
}

$\mathrm{A}_{\mathrm{h}}^{36}$ 36-year-old previously healthy man from southwestern Ontario presented to the emergency department with an episode of cough producing $50 \mathrm{~mL}$ of bright red blood after a 2-week history of coughing up green sputum. He was otherwise asymptomatic, and his physical examination was unremarkable. A chest radiograph showed mild airspace disease at the left lung base. A CT scan of the thorax with intravenous contrast medium revealed narrowing of the left descending pulmonary artery and an increase in soft tissue in the mediastinum, with extensive calcification in the subcarinal and left hilar region (Fig. 1, white arrows). This soft tissue extended posterior to the left atrium and along the left posterior mediastinum (Fig. 2, black arrows). The right inferior pulmonary vein was normal (Fig. 2, arrowhead), but the left inferior pulmonary vein was unopacified, indicating occlusion (Fig. 2, white arrow). Interlobular septal thickening due to venous obstruction (image not shown) within the left lower lobe was seen. The findings were pathognomonic of fibrosing mediastinitis. Bronchoscopy with bronchoalveolar lavage was performed to rule out other causes of hemoptysis. The patient was otherwise well and was discharged home, but 1 month later he reported mild dyspnea and chest discomfort, and he was given prednisone at $0.5 \mathrm{mg} / \mathrm{kg}$ daily. His symptoms improved over the next month.

Fibrosing mediastinitis is characterized by the proliferation of fibrous tissue in the mediastinum, which usually arises as a rare ( $<1 \%)$, late complication of Histoplasma capsulatum infection in regions where the fungus is endemic. ${ }^{1,2}$ In North America, these regions include the St. Lawrence River valley, southwestern Ontario, and the central, midAtlantic and southeastern states,

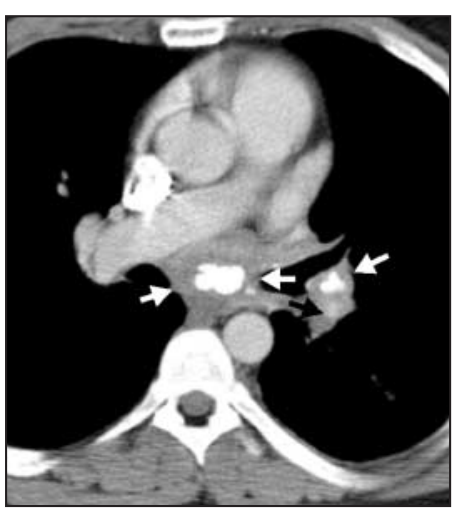

particularly in the Ohio and Mississippi River valleys. Because the fungus is common in soils contaminated with bat or bird droppings, people who work with soil are at increased risk for histoplasmosis. Immunocompromised people are at particular risk for disseminated infection. Fibrosing mediastinitis fibrosis appears to arise when fungal antigen spills out of mediastinal lymph nodes and precipitates an extensive fibrotic immune response, resulting in encasement of mediastinal structures. ${ }^{2}$ Risk factors for the disease are uncertain, although male predilection, an association with idiopathic retroperitoneal fibrosis and a correlation with human leukocyte antigen-A2 have been reported. ${ }^{3}$ The most common manifestation is superior vena cava syndrome, which leads to dyspnea, facial plethora and neck distention. Compression of the bronchopulmonary tree may cause obstructive respiratory symptoms and infections, and esophageal involvement may lead to dysphagia. Pulmonary venous involvement may cause pulmonary edema, resulting in interlobular septal thickening, as seen in our patient. Hemoptysis may result from postobstructive necrotizing pneumonia, pulmonary venous hypertension or the development of fistulas between the pulmonary and bronchial arterial circulations. Mortality has been reported to be as high as $30 \%$. $^{1}$

Diagnosis usually rests on a combination of typical clinical

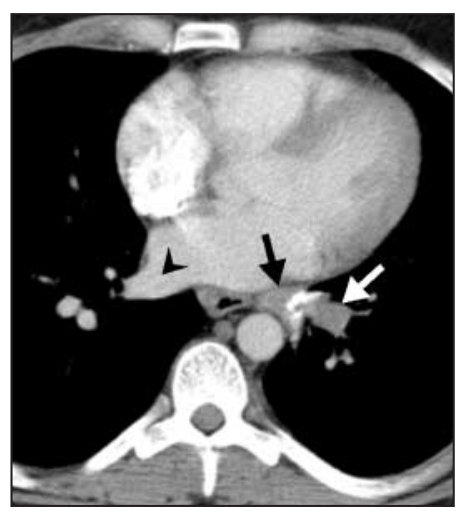

and radiographic findings on $\mathrm{CT}$ or MRI. Biopsy may be required in areas where Histoplasma is not endemic. Tissue culture is typically sterile. Serologic studies and fungal antigen testing for $M$ and $\mathrm{H}$ bands are generally not helpful, since these tests are useful only for diagnosing acute infection. Antifungal agents and corticosteroids have been used in some cases, but there is little evidence of clinical benefit and these agents are generally not recommended unless there is active infection or inflammation. ${ }^{4}$ Surgery may be indicated for decompression of airway, vascular or esophageal obstruction.

\section{Darrell Tan}

Department of Internal Medicine Michael Hutcheon

Division of Respiratory Medicine

University Health Network

Toronto General Hospital

Tae Bong Chung

Department of Medical Imaging

University of Toronto

University Health Network and Mount Sinai Hospital

Toronto, Ont.

\section{References}

1. Loyd JE, Tillman BF, Atkinson JB, Des Prez RM. Mediastinal fibrosis complicating histoplasmosis. Medicine (Baltimore) 1988;67:295-310.

2. Wheat LJ. Histoplasmosis susceptibility in humans. In: Jacobs PH, Nall L, editors. Fungal disease. New York: Marcel Dekker; 1997. p. 239.

3. Peebles RS, Carpenter CT, Dupont WD, Loyd JE. Mediastinal fibrosis is associated with human leukocyte antigen-A2. Chest 2000;117:482-5.

4. Wheat J, Sarosi G, McKinsey D, Hamill R, Bradsher R, Johnson P, et al. Practice guidelines for the management of patients with histoplasmosis. Clin Inf Dis 2000;30:688-95. 